\title{
VACCINIA GANGRENOSA THE REPORT OF A CASE OF PROLONGED GENERALIZED VACCINIA
}

\author{
BY \\ BERNARD LAURANCE, A. C. CUNLIFFE and J. A. DUDGEON \\ From King's College Hospital, London, and the Virus Laboratory, The Hospital for Sick Children, Great Ormond \\ Street, London
}

(RECEIVED FOR PUBLICATION FEBRUARY 28, 1952)

Serious complications of Jennerian vaccination are extremely rare. The fact, however, that they may occur and endanger life warrants a careful enquiry into any such incident.

The more common complications are bacterial infection of the vaccination site and non-specific rashes of erythema multiforme, morbilliform and urticarial types; except for tetanus, which is becoming exceedingly rare, these are seldom dangerous. The most serious complications are post-vaccinial encephalitis and generalized vaccinia.

Generalized vaccinia is characterized by the appearance within six to fourteen days after vaccination of secondary vaccinial lesions varying in number from a few isolated vesicles to an extensive eruption covering large areas of skin, and presumably resulting from dissemination of virus by the blood stream. The secondary lesions mature in the same way as, but more rapidly than, the primary lesion, so that characteristically both primary and secondary lesions regress simultaneously. In patients with healthy intact skin a rapid recovery is usual and the majority, if not all of the secondary lesions, unlike the primary, resolve without obvious scarring.

The course of this disorder in patients with a preexisting skin lesion such as eczema, seborrhoeic dermatitis or a burn is very different. The possibility of generalized vaccinia is greater in such cases and it is usually accompanied by severe toxaemia. The mortality rate may be as high as $25 \%$ (Ormsby and Montgomery, 1948), though recent figures suggest that the mortality is now much lower (Fries, Borne and Barnes, 1948). Patients with skin diseases are also more liable to develop generalized vaccinia as a result of accidental or auto-inoculation. Thus following the extensive vaccination campaign in New York City in 1947, Greenberg (1948) found that 38 out of 45 cases of generalized vaccinia occurred in persons with preexisting skin disease and furthermore, that 28 out of these 38 patients had not been vaccinated, but were presumed contacts of recently vaccinated persons.

The true incidence of generalized vaccinia is difficult to assess, since accurate information as to the total number of vaccinations performed is seldom available, whereas complications are usually carefully recorded. Jubb (1943) states that most estimates of the incidence of generalized vaccinia vary from 1 in 10,000 to 1 in 100,000 . Gins (1930) records an incidence of slightly less than 1 in $1,000,000$ in Germany. Probably the most accurate figures available in this country are contained in the reports of the Chief. Medical Officer to the Ministry of Health for the years 1949 and 1950 where there are recorded 15 cases of generalized vaccinia amongst 373,766 infants vaccinated in the first year of life, an incidence of approximately 1 in 25,000 . No deaths occurred.

The case to be described in this paper differs from the typical form of generalized vaccinia in three respects ; first, in the prolonged course of the illness-secondary vesicles appeared intermittently until death 83 days after vaccination ; secondly, in the ulceration of both primary and secondary lesions ; and thirdly in the apparent failure of specific antibody formation.

The clinical history and course of the illness in this child resembles the condition first described by Dr. Jonathan Hutchinson in 1879 as vaccinia gangraenosa and reported by him in 1882 .

\section{Case Report}

We are indebted to the patient's family doctor for the following history up to the time of admission to hospital.

The patient, a girl, was born at full term on February 15, 1951, after a normal pregnancy and delivery. The birth weight was $7 \mathrm{lb} .8 \mathrm{oz}$. She was entirely breast fed for 10 weeks and subsequently these feeds were complemented with tuberculin-tested cow's milk.

At the age of 3 weeks she developed an axillary abscess following intertrigo at the same time as the mother had a breast abscess. Both lesions healed uneventfully. 
Her 2-year-old sister had reacted normally to primary vaccination in infancy; in both parents reaction to primary and secondary vaccination had been uncomplicated.

On June 12 the patient (aged 4 months) was vaccinated. Two 1 inch parallel scratches were made through the lymph on the left upper arm and a boric lint dressing was applied. No blood was drawn. The following day a mild erythematous rash appeared on the trunk which subsided in one week, though at this time the parents thought she was 'feverish and ill'. By the seventh day a papule had developed at the site of vaccination and a fresh boric lint dressing was applied. On the fourteenth day the vaccinated area was found to be moist and enlarging and a second vesicle appeared which later ulcerated and coalesced with the primary lesion. The dressing was removed and the area dusted with sterile talcum powder. About 21 days after vaccination some small vesicles were present in the groins and pubic region ; these also ulcerated. Fresh crops continued to appear at about two-weekly intervals in different areas of the body.

On August 15, 64 days after vaccination, because the lesions were extending, the child (aged 6 months) was admitted to King's College Hospital under the care of Dr. Mary Wilmers.

She had had an infrequent non-productive cough which had become worse for the week before admission, and which was followed by regurgitation and occasionally by vomiting. Her bowel action was regular with normal motions and micturition was normal.

When examined on admission, the child looked ill, pale and listless, but showed no signs of dehydration. The temperature was $104 \cdot 8^{\circ} \mathrm{F}$., the pulse rate 154 and the respirations 38 per minute. She weighed $12 \mathrm{lb} .2 \mathrm{oz}$. Multiple small, shallow, circular ulcers $\frac{1}{2}$ to $3 \frac{1}{2}$ in. in diameter were present in the following situations: primary vaccination site of the left upper arm; inner aspect of the left elbow ; inner aspect of the left thigh and left calf ; right calf ; both groins ; buttocks and part of the vulvae ; left side of the nose ; the right pinna. They all had clear-cut shelving, non-indurated edges with granulating bases (Fig. 1). There was one umbilicated vesicle, $\frac{1}{2}$ in. in diameter, on the right calf and several scattered erythematous areas. No enlarged glands were found.

Breath sounds were harsh and scattered rhonchi were heard throughout both lung fields. The cardiovascular and central nervous systems were normal. There was a

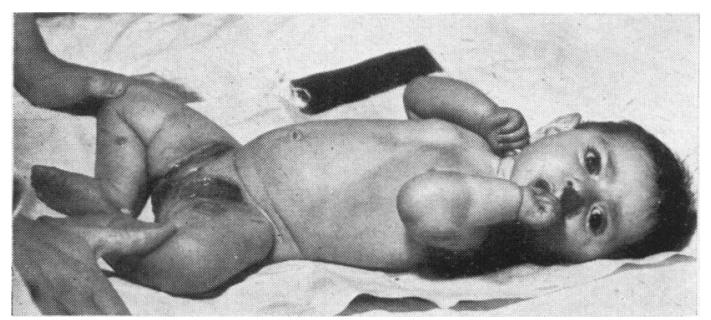

FIG. 1.-Appearance of the patient on admission. Note ulcer of the nose and a vesicle on the right calf. severe thrush infection in the mouth, but otherwise the digestive system was normal.

Results of investigations at this time were : white cells 17,500 per c.mm. (neutrophils $85 \%$, lymphocytes $10 \%$, monocytes $4 \%$ and eosinophils $1 \%$ ). No abnormality was seen in a radiograph of the chest. Candida albicans and haemolytic streptococci (Lancefield Group G) were cultured from the lesions of the throat and mouth, and Staphylococcus pyogenes from those of the nose and ear. From the vaccination site and other ulcers a mixed growth of Staph. pyogenes, coliform bacilli, haemolytic streptococci (Lancefield Group G) and Candida albicans was cultured. Fluid from the vesicle on the right calf gave a growth of haemolytic streptococci (Lancefield Group G).

On admission a tentative diagnosis of ecthyma was made. Generalized vaccinia was considered unlikely because of the long duration of the disease. As the illness progressed the diagnosis of generalized vaccinia had to be reconsidered, particularly in view of the similarity of this case to one of proved vaccinial aetiology seen in 1948 under the care of Dr. H. J. Wallace at St. Thomas's Hospital, London.

The thrush infection in the mouth improved rapidly after treatment with $0.5 \%$ aqueous gentian violet. Later, because of the bacterial infection of the skin, intramuscular penicillin $(20,000$ units four-hourly) and oral chloramphenicol $(80 \mathrm{mg}$. q.d.s.) were given for 15 days. Lotio hydrargyri perchloridi was applied locally and vitamins $\mathrm{A}, \mathrm{B}, \mathrm{C}$ and $\mathrm{D}$ were given by mouth. This treatment appeared to control the infection for a short time, but later the ulcers enlarged and fresh lesions appeared, developing from papules to umbilicated vesicles within 24 to 48 hours. Four to six days later these ruptured, becoming ulcers without any intervening pustular stage.

On August 21, local treatment was changed to gentian violet $(1 \%)$ in $50 \%$ Lassar's paste. Two days later raised, whitish, granulomatous lesions were seen in the mouth. The abnormal chest signs had now cleared, but the general state of the child remained unchanged.

By August 25 her condition had deteriorated, with sudden attacks of pallor and distressed, rapid respirations. The ulcers were increasing in size and new vesicles appeared almost daily (Fig. 2). The spleen was now palpable. Aureomycin powder was insufflated on to the ulcers, but had no apparent effect.

On August 31, the child's condition was worse. Oral chloramphenicol and local aureomycin were discontinued and tyrothricin ointment was applied to the ulcers. Cultures from ulcers in the groins yielded a predominant growth of $P$ s. pyocyanea.

She remained very ill and died on September 3, 83 days after primary vaccination.

Fig. 3 shows the course of the illness in this child compared with the normal course following Jennerian vaccination.

\section{Virus Investigation}

Some vesicle fluid and a few smears were taken on August 31, but most of the material was collected a few hours after death. The pathological findings will be 


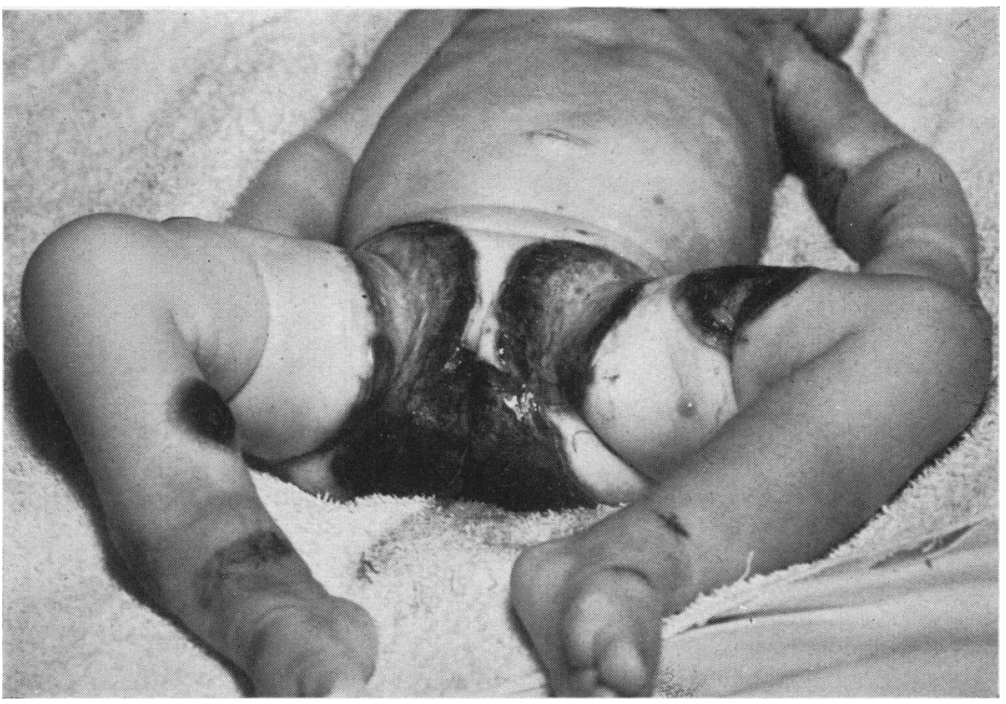

FIG. 2.-Appearances at death.

fully reported by Hall, Cunliffe and Dudgeon; the following is a summary of their essential findings.

Direct Examination. Smears of vesicle fluid showed a few necrotic polymorphs, Gram-positive cocci and Gramnegative rods. A few clumps of minute organisms morphologically similar to vaccinia elementary bodies were also seen but not in sufficient numbers to make a diagnosis.

Isolation. Vesicle fluid, in which the growth of $P s$. pyocyanea was controlled by the addition of chloramphenicol before inoculation, produced confluent lesions on the chorio-allantois of the chick-embryo and severe kerato-conjunctivitis in the rabbit.

The blood (serum in this case) and selected material from the liver, spleen and bone-marrow gave rise to similar but less numerous lesions on the chorio-allantois, careful precautions having been taken to avoid contamination with virus from the ulcerated areas of skin.

Serology. Vesicle fluid produced positive complement fixation to a titre of 1 in 160 with hyperimmune vaccinial serum.

Serum (inactivated) taken on September 3 showed no demonstrable antibody when tested by the neutralization, complement-fixation and haemagglutinin-inhibition tests.

Virus Identification. The virus was identified as vaccinia on the following grounds: (1) the positive complement fixation of the vesicle fluid with vaccinia antiserum ; (2) the microscopic appearance of the chorio-allantoic. lesions and the presence of intracytoplasmic inclusions; (3) the neutralization of the virus by vaccinial antiserum.

\section{Necropsy}

Dr. G. F. M. Hall performed the necropsy.

The body was that of a fairly well-nourished female infant with numerous ulcerated skin lesions. There were also two small vesicles, one on the abdomen and the other on the left thigh. Another recent vesicle was found on the dorsum of the tơngue. Several patches of thrush were present on the palate and pharynx.

Both lungs were partially collapsed and contained numerous scattered, well-defined white areas, averaging 1 to $2 \mathrm{~mm}$. in diameter. These areas were surrounded by a narrow haemorrhagic zone. Similar white foci were present in large numbers in the liver and in moderate numbers in the kidneys. The liver showed marked fatty change. The spleen was slightly enlarged but was otherwise macroscopically normal. Permission was not received for examination of the brain.

Sections of the recent vesicles on the skin and tongue showed areas of necrosis with complete absence of cellular reaction. The epithelium at the edges of the lesions was somewhat oedematous and degenerate. Section of the ulcer at the site of vaccination showed an essentially similar appearance, except that the necrotic zone was denser and extended deeper.

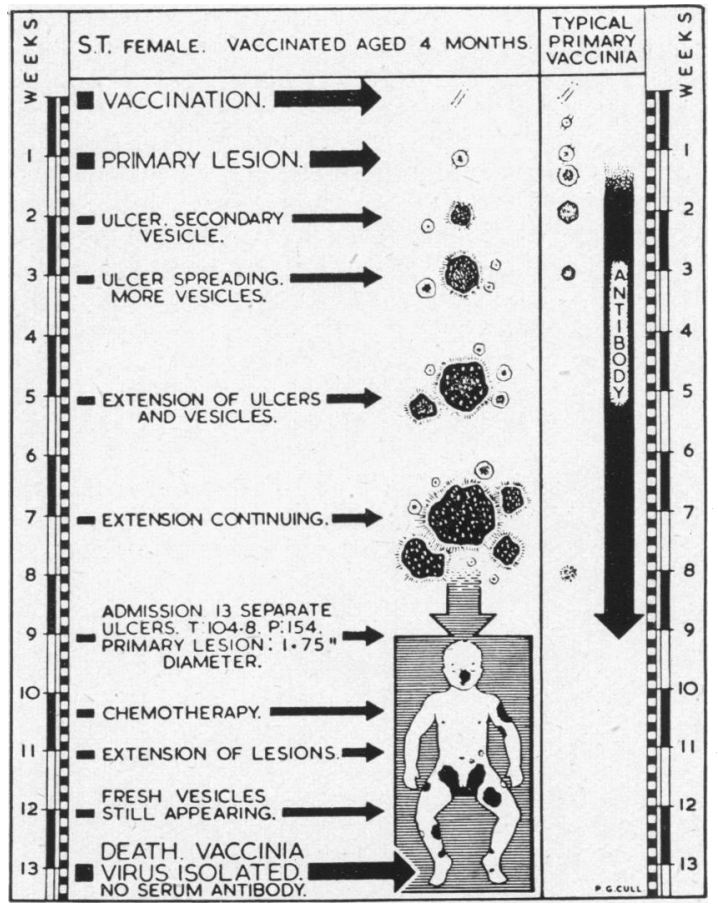

FIG. 3.-Diagram illustrating the course of the illness compared with the normal course following Jennerian vaccination. 
Sections of the white areas in the lungs, liver and kidneys showed these to be well-defined foci of recent coagulative necrosis with no leucocytic infiltration. Similar, but microscopic, foci of necrosis were found in both adrenal glands.

Large numbers of Gram-negative bacilli were present in the necrotic foci in the internal organs and tongue, and at the necrotic edges of the skin lesions, both recent and old. No inclusion bodies were demonstrated.

\section{Discussion}

Although the time between the appearance of the lesions and the vaccination of this infant was known when she was admitted to hospital, the diagnosis of generalized vaccinia was not then seriously entertained because of the length of the history and the ulcerative nature of the lesions ; a disorder of bacterial origin, such as ecthyma, was thought to be more likely, However, although the ulcers appeared cleaner after local and systemic chemotherapy, no obvious healing took place. Anaerobic streptococci are often credited with the production of intractable infections of the skin; in this case, however, there was no bacteriological support for this hypothesis. Streptococci of Lancefield Group $G$ are occasionally associated with severe ulcerative skin lesions (Fry, personal communication, 1952); in this patient however, the lesions progressed in spite of the elimination of the streptococci by chemotherapy.

As the disease progressed it became increasingly evident that this illness might well be due to a persistent vaccinial infection. This tentative clinical diagnosis was confirmed by isolation of vaccinia virus from several cutaneous lesions, as well as from the heart blood, liver, spleen and bone marrow at necropsy. This child died, therefore, as the result of prolonged generalized vaccinia.

In the early days of Jennerian vaccination various ulcerative conditions were amongst the wellrecognized complications of vaccination. Ulceration was most commonly confined to the area around the inoculation site, but occasionally the lesions were widespread. Hutchinson (1882) described the death of a child from a generalized eruption 20 days after vaccination, in which the lesions became gangrenous. He applied the term 'vaccinia gangraenosa' to his case and to that reported in Ireland by Stokes (1880). In both these cases gangrene occurred at the site of the secondary lesions only; the vaccination area was not involved. In Stokes's case the history of vaccination was obviously unreliable and gross secondary infection was probable. Hutchinson held that in both the lesions were directly due to normal calf lymph virus. Crocker (1887, 1903) maintained that these ulcerative lesions were mainly of bacterial origin and he included under the term 'dermatitis gangraenosa infantum' the gangrenous complications of vaccinia with those of other acute exanthems, such as varicella, as well as with less specific conditions such as cancrum oris. (It is interesting to note that he suspected Ps. pyocyanea of playing an important part in these infections, since in four of the cases mentioned below this organism was present.) Whether Hutchinson's and Stokes's cases are the same as ours is debatable, for at that time vaccine lymph was not standardized; arm to arm vaccination was still frequently practised and the risks of sepsis were vastly greater than to-day. The fact remains, however, that it was obviously Hutchinson's view that both cases were atypical forms of generalized vaccinia.

Undoubted examples of prolonged generalized vaccinia have been reported by Acland (1906) and Bigler and Slotkowski (1951). Acland's case occurred in 1892 in an infant aged 3 months in whom ulceration of both primary and secondary lesions continued until death, eight weeks after vaccination. Evidence of the vaccinial origin of these lesions is suggested by the fact that a few days before the child's death the mother developed a vaccinial lesion on the breast. The case reported by Bigler and Slotkowski (1951) followed vaccination of a negro child at the age of 5 years. The lesions were more extensive than in our case and at death, after four months' illness, cytoplasmic inclusions were demonstrated in the cutaneous lesions. In 1938 Conybeare on behalf of the Ministry of Health investigated a case of generalized vaccinia associated with primary vaccination in a man aged 31 in whom death occurred after 10 months following very extensive and intractable ulceration. Another case of prolonged generalized vaccinia was seen by Crosbie and Downie in 1943, in which a girl, vaccinated at the age of 11 months, developed vesicles during the following two months. Ulceration, although widespread, was largely confined to the vaccinated area. Although the lesions were healing, death occurred from an intercurrent infection. No vaccinial antigen or antibody was detected in blood taken at necropsy. In Dr. Wallace's patient, to whom reference has already been made, ulceration was similar in extent to that in our case and the illness lasted four and a half months; the diagnosis was confirmed by the isolation of the virus.

As only six cases of prolonged generalized vaccinia have come to our notice, the condition is obviously very rare and the mortality high ; death occurred in five of these six cases. It seems pertinent to enquire how this condition differs 
from primary and from typical generalized vaccinia, and what is the probable cause. In none of the cases mentioned above was there any evidence that the lymph was at fault; in the present case Lister Institute vaccine lymph was used and 49,050 doses of the same batch were issued to more than two dozen public health laboratories without the report of any untoward reactions. It is now generally accepted that in primary vaccinia virus multiplies locally at the site of inoculation, enters the bloodstream following this multiplication and disappears from the circulation as antibody appears towards the end of the first week. In generalized vaccinia an extension of this process occurs; virus, which may be disseminated over a wide area of skin, multiplies at these sites and gives rise to the secondary vaccinial rash. This condition generally runs a limited course, provided the child does not succumb to toxaemia, which is more likely to occur in such conditions as eczema ; recovery with healing is usual. It seems probable that virus multiplication in this child was largely confined to the skin rather than to the internal organs, which is in agreement with the views of Fenner (1948a and b) on the pathogenesis of generalized vaccinia. In our case, there was neither evidence of healing, nor sign of a tissue response to infection. This was associated with an absence of demonstrable antibody formation.

Some of the differences between primary vaccinia, typical generalized vaccinia and this prolonged form can probably be attributed to variation in the immunity mechanism. In typical generalized vaccinia there is evidence that antibody production is normal, and it has been suggested that local cutaneous immunity may be defective (Evans, 1937). Bigler and Slotkowski (1951) quote a case in which antibody production was delayed. In at least two of the cases of prolonged generalized vaccinia the failure of vaccinial antibody formation was complete. The reasons for this failure, whether total or specific, must be purely conjectural ; it is interesting to note, however, that when this child was aged 3 weeks, an axillary abscess, probably staphylococcal, did not generalize. This suggests that the child possessed some powers of resistance. On the other hand it might well be suggested that such an infection would not have occurred in a child with normal immunity.

Treatment. There is no doubt that the previous high mortality rate of generalized vaccinia in eczematous patients and of generalized herpes Kaposi's varicelliform eruption was to a great extent due to the secondary infection and in both these conditions local and systemic chemotherapy has been used with success. No antibiotic, however, has yet been shown to have any effect on either the vaccinia or herpes virus.

On theoretical grounds it would seem desirable to supplement chemotherapy by giving hyperimmune vaccinial serum parenterally to replace deficient antibody. There is, however, little evidence of the efficacy of antiserum in generalized vaccinia in man ; in the case seen by Crosbie and Downie, to which reference has been made, it is possibly significant that after the administration of serum from a recently vaccinated adult healing, as shown by the appearance of granulation tissue at the base of the ulcers, was noted for the first time. In the absence of hyperimmune serum, serum from a recently vaccinated person or possibly gamma globulin containing vaccinia virus antibodies might be used in liberal dosage.

\section{Summary}

A fatal case of generalized vaccinia is described in which ulceration of the primary and secondary lesions continued for nearly three months. The patient's serum contained no demonstrable vaccinial antibodies. Vaccinia virus was isolated from the skin lesions, blood, liver and spleen.

The literature is reviewed and the relation of vaccinia gangrenosa to typical generalized vaccinia is discussed.

We are indebted to Dr. Mary Wilmers for permission to publish this case, and we wish to express our thanks to her and to Dr. Wilfrid Sheldon, Dr. David Williams and Professor Magnus for much helpful advice; to Dr. Hugh Wallace for kindly allowing us access to the notes of the patient seen at St. Thomas's Hospital and permission to publish; and to Dr. F. O. MacCallum, who isolated the virus in the latter case, Professor A. W. Downie, Dr. E. T. Conybeare and Dr. Douglas McClean for much advice and information.

The photographic department at King's College Hospital kindly supplied the photographs.

REFERENCES

Acland, T. D. (1906). In Allbutt and Rolleston's System of Medicine. vol. 2, part 1, p. 689 .

Bigler, J. A. and Slotkowski, E. L. (1951). Pediatrics, 7, 24

Crocker, H. Radcliffe (1887). Brit. med. J., 1, 1333 . Evans, P. R. (1937). Archives of Disease in Childhood, 12, 333.

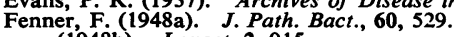

Fenner, (1948b). Lancet, 2, 915.

Fry, R. M. (1952). Personal communication.

Fries, J. H., Borne, S. and Barnes, H. L. (1948). J. Pediat., 32, 532

Gins, H. A.' (1930). Zbl. ges. Kinderheilk., 24, 145.

Greenberg, M. (1948). Amer. J. Dis. Child., 76, 492

Hutchinson, J. (1882). Amed.-chir. Trans., 65 (2 ser. 47), 1.

Jubb, A. A. (1943). Brit med. J., 1, 91. Ormsby, O. S. and Montgomery, H. (1948). Diseases of the Skin,

Reports of the Chief Medical Officer of Health (1949-50). Part II, 26.

Ibid. (1950-51). Part II, 24.

Stokes, W. (1880). Dublin J. med. Sci., 69, 497. 